\title{
Atuação do fisioterapeuta intensivista durante a pandemia de COVID-19: Desafios e modificações na prática clínica
}

\section{Performance of the intensivist physiotherapist during the COVID-19 pandemic: Challenges and improvements in clinical practice}

\author{
Caroline de Oliveira Viana ${ }^{1}$ (1) \\ Carla Mônica Nunes Pombo² \\ Márcia Cardinalle Correia Viana ${ }^{3}$ (1)
}

\author{
Marcus César Silva de Morais 4 (1) \\ Márcia Maria Pinheiro Dantas 5 (1)
}

\begin{abstract}
1'Autora para correspondência. Centro Universitário Christus (Fortaleza). Ceará, Brasil. vianacaroline5@gmail.com 2,3 Hospital Geral Dr. César Cals (Fortaleza). Ceará, Brasil. carlamonicapombo.cmp@gmail.com, mccviana@outlook.com

4,5Instituto Doutor José Frota (Fortaleza). Ceará, Brasil. moraismarcus@uol.com.br, marciapdantas759@gmail.com
\end{abstract}

RESUMO I INTRODUÇÃO: A doença causada pelo Novo Coronavírus (SARS-CoV-2), gerou um fluxo crescente de pacientes infectados que necessitaram de atenção especializada em terapia intensiva, impactando sobremaneira o Sistema de Saúde, repercutindo de forma direta na experiência de Fisioterapeutas Intensivistas, profissionais fundamentais nessa assistência. OBJETIVO: Verificar modificações na prática clínica do fisioterapeuta intensivista durante a Pandemia de COVID-19 e identificar desafios enfrentados durante assistência aos pacientes infectados. MATERIAIS E MÉTODOS: Trata-se de um estudo de campo, exploratório, transversal e quantitativo, realizado de fevereiro a abril de 2021, com fisioterapeutas intensivistas de dois hospitais públicos (um municipal e outro estadual) da cidade de Fortaleza. Foi realizado um cálculo do fluxo de fisioterapeutas atuantes nessas unidades, estimando-se uma amostra de 71 profissionais para compor o estudo. Participaram da pesquisa os Fisioterapeutas Intensivistas que realizaram assistência a pacientes com COVID-19 e que possuíam vínculo com os referidos hospitais. Foram excluídos os fisioterapeutas na função de residentes, estagiários e preceptores presentes. Para coleta de dados utilizou-se formulário on-line viabilizado pela plataforma Google Forms, composto por perguntas objetivas relacionadas aos dados do profissional, infecção destes por COVID-19, presença de fatores de risco, modificações na prática clínica durante assistência, desafios enfrentados, situações vivenciadas e segurança durante atendimento ao paciente crítico com COVID-19. Os dados foram tabulados através do Software Statistical Package for the Social Sciences (SPSS), versão 20.0. Utilizou-se estatística descritiva com frequências absolutas e relativas. RESULTADOS: Participaram do estudo 59 fisioterapeutas intensivistas atuantes na linha de frente contra a COVID-19; destes, 40,6\% foram infectados pelo vírus SARS-CoV-2. As principais modificações relatadas foram melhora no manejo da ventilação mecânica $(86,4 \%)$ e melhor integração com equipe multidisciplinar (62,7\%). Dentre os desafios enfrentados durante a assistência, o contexto completamente novo $(89,9 \%)$ e o testemunho de experiências dolorosas de pacientes $(76,3 \%)$ foram os mais marcantes. CONCLUSÃo: A pandemia proporcionou aos fisioterapeutas intensivistas novos desafios e modificações na prática clínica, requerendo atualização, implantação de novas práticas e maior interação em equipe multidisciplinar.

PALAVRAS-CHAVE: Fisioterapeutas. Unidades de Terapia Intensiva. COVID-19.
ABSTRACT | INTRODUCTION: The disease caused by the New Coronavirus (SARS-CoV-2) generated a growing flow of infected patients who needed specialized care in intensive care, greatly impacting the Health System, directly impacting the experience of Intensive Care Physiotherapists fundamental professionals in this assistance. OBJECTIVE: To verify changes in the clinical practice of the intensive care physical therapist during the COVID-19 Pandemic and to identify challenges faced during the care of infected patients. MATERIALS AND METHODS: That is an exploratory, transversal, and quantitative field research carried out from February to April 2021, with intensive care physical therapists from two public hospitals (one municipal and one state) in the city of Fortaleza. A calculation of the flow of physiotherapists working in these units was performed, estimating a sample of 71 professionals to compose the study. The Intensivist Physiotherapists who helped patients with COVID-19 and linked with the hospitals participated in the research. Physiotherapists in the role of residents, trainees, and preceptors present were excluded. For data collection, an online form made possible by the Google Forms platform was used, composed of objective questions related to the professional's data, their infection by COVID-19, presence of risk factors, changes in clinical practice during care, challenges faced, situations experienced and safety during critical patient care with COVID-19. Data were tabulated using the Software Statistical Package for the Social Sciences (SPSS), version 20.0. Descriptive statistics with absolute and relative frequencies were used. RESULTS: 59 intensive care physical therapists working on the front line against COVID-19 participated in the study; of these, $40.6 \%$ were infected with the SARS-CoV-2 virus. Improvement in the management of mechanical ventilation (86.4\%) and better integration with the multidisciplinary team $(62.7 \%)$ were the main changes reported. Among the challenges faced during care, the completely new context (89.9\%) and witnessing painful experiences of patients (76.3\%) were the most striking. CONCLUSION: The Pandemic provided intensive care physical therapists with new challenges and changes in clinical practice, requiring updating, implementation of new practices, and greater interaction in a multidisciplinary team.

KEYWORDS: Elderly. COVID-19. Quality of life. Functionality. Physiotherapy. Post-covid-19. 


\section{Introdução}

A doença causada pelo novo Coronavírus (COVID-19) foi identificada, inicialmente, em dezembro de 2019 na cidade de Wuhan (província de Hubei, China). ${ }^{1}$ Após o primeiro relato, em menos de quatro meses disseminou-se em todo o mundo, levando o Comitê de Emergência da Organização Mundial da Saúde (OMS) a declarar uma Emergência de Saúde Pública a nível Internacional em 30 de janeiro de 2020, e como uma situação pandêmica, em 11 de março de 2020.2.3.

Pessoas acometidas por esta doença apresentam sintomas heterogêneos, variando desde infecções assintomáticas a quadros graves, sendo que a literatura destaca os principais: febre (89\%), tosse (68\%), fadiga (38\%), produção de secreções (34\%) e falta de ar (19\%)., Essencial ressalvar que a gravidade da doença apresenta intrínseca relação com a presença de condições prévias, como obesidade, idade avançada e algumas comorbidades. ${ }^{6,7}$ De acordo com a Organização Mundial de Saúde (OMS), $80 \%$ dos indivíduos infectados apresentam-se assintomáticos ou oligossintomáticos, 15\% desenvolvem os sintomas graves, e uma porcentagem menor se apresenta de forma crítica. . $^{8}$

As Unidades de Terapia Intensiva (UTI's) apresentam o propósito principal de oferecer atenção contínua e suporte avançado aos pacientes críticos com risco de morte. ${ }^{3}$ No caso da COVID-19, o fluxo crescente de pacientes críticos neste ambiente que necessitam de maior atenção especializada aumenta o risco de colapso do sistema de saúde que, de uma forma geral, encontra-se sobrecarregado.2.9 Neste contexto, a equipe multiprofissional de saúde é de suma importância no atual cenário pandêmico e, por volta da década de 70, o fisioterapeuta foi inserido nestas equipes, evoluindo rapidamente e passando por processo de subdivisão em especialidades, como a Fisioterapia em Terapia Intensiva, que foi regulamentada pela Resolução n. 402/2011, do Conselho Federal de Fisioterapia e Terapia Ocupacional (COFFITO). 5,10,11,12

A pandemia de COVID-19 repercutiu de forma direta na experiência desses profissionais, e a busca por conhecimento está a todo o momento sendo construída e reconstruída, exigindo que o profissional se mantenha sempre atualizado, repensando o trabalho em equipe como um todo e implementando novas condutas terapêuticas. ${ }^{9,13}$ Estes profissionais no atual cenário enfrentam uma excessiva carga de trabalho, gerando uma grande preocupação quanto à manutenção da qualidade dos processos assistenciais..,14

Diante disso, o objetivo desse estudo foi verificar as modificações na prática clínica de Fisioterapeutas Intensivistas durante a Pandemia de COVID-19, bem como identificar os desafios enfrentados para assistência à população de pacientes críticos infectados pelo vírus SARS-CoV-2.

\section{Materiais e métodos}

Trata-se de uma pesquisa de campo, exploratória, transversal e com abordagem quantitativa, realizada no período de fevereiro a abril de 2021, com Fisioterapeutas Intensivistas atuantes na linha de frente no combate ao COVID-19 em dois Hospitais públicos na cidade de Fortaleza, sendo um da rede estadual e o outro municipal, ambos referência na assistência ao paciente com COVID-19.

O estudo teve a aprovação do Comitê de Ética em Pesquisa dos Hospitais públicos mencionados com os pareceres $n^{\circ} 4.476 .987$ e $n^{\circ} 4.578 .191$. As normas e diretrizes da Resolução 466/12 do Conselho Nacional de Saúde/Ministério da Saúde foram obedecidas. $\frac{15}{}$ Foi realizado um cálculo do fluxo de fisioterapeutas atuantes nessas unidades, estimando-se uma amostra de 71 profissionais para compor o estudo. Participaram da pesquisa os Fisioterapeutas Intensivistas que realizaram assistência a pacientes com COVID-19 e que possuíam vínculo com os referidos hospitais. Foram excluídos os fisioterapeutas na função de residentes, estagiários e preceptores presentes.

A coleta de dados foi realizada por meio de um questionário elaborado pelas pesquisadoras, baseado em leitura de artigos prévios sobre a temática em questão.2,14,16,17 Foi composto por perguntas objetivas relacionadas aos dados do profissional, infecção destes por COVID-19, presença de fatores de risco, modificações na prática clínica durante assistência, desafios enfrentados, situações vivenciadas e segurança durante atendimento ao paciente crítico com COVID-19. 
Por conta da Pandemia de COVID-19 e os riscos eminentes de contaminação, a pesquisa ocorreu de forma virtual, por meio da plataforma on-line, Google Forms. Os profissionais foram convidados a participar da pesquisa por meio de convites encaminhados aos chefes de serviço, sendo disponibilizado o link (https://forms.gle/TcB1UFkdAkweGFcx8) através dos grupos de WhatsApp dos seus respectivos Hospitais. O Termo de Consentimento Livre e Esclarecido (TCLE) foi disponibilizado on-line na página inicial e o participante só teve acesso ao instrumento de coleta de dados caso concordasse em participar da pesquisa. Os participantes tiveram dez dias a partir do envio do questionário para respondê-lo de forma anônima e voluntária.

Os dados coletados foram inicialmente tabulados no software Excel 2017 e então transferidos para o software estatístico SPSS (Statistical Package for the Social Sciences) versão 20.0. Utilizou-se a estatística descritiva com frequências absolutas e relativas, com resultados demonstrados através de figuras e tabelas.

\section{Resultados}

Participaram do estudo 59 Fisioterapeutas intensivistas, 49 (83,0\%) do gênero feminino. Dos profissionais participantes, $42(71,1 \%)$ são da rede municipal. Na Tabela 1 estão expostos dados referentes ao tempo de formação e de atuação em UTI, bem como o nível máximo de titulação e a quantidade de UTI em que atuam.

Tabela 1. Tempo de formação e atuação em UTI, nível máximo de titulação e quantidade de UTI's em que atuam. N=59

\begin{tabular}{|c|c|c|c|}
\hline & \multicolumn{2}{|c|}{ Hospital } & \multirow[b]{2}{*}{ Total N(\% } \\
\hline & Municipal & Estadual & \\
\hline \multicolumn{4}{|l|}{ Tempo de formado } \\
\hline 1 a 4 anos & $4(9.5 \%)$ & $6(35.3 \%)$ & $10(16.9 \%)$ \\
\hline 5 a 10 anos & $17(40.5 \%)$ & $4(23.5 \%)$ & $21(35.6 \%)$ \\
\hline 11 a 15 anos & $9(21.4 \%)$ & $2(11.8 \%)$ & $11(18.6 \%)$ \\
\hline 16 a 20 anos & $6(14.3 \%)$ & $0(0.0 \%)$ & $6(10.2 \%)$ \\
\hline Mais de 20 anos & $6(14.3 \%)$ & $5(29.4 \%)$ & $11(18.6 \%)$ \\
\hline \multicolumn{4}{|l|}{ Tempo de atuação em UTI } \\
\hline Ingressou durante a Pandemia & $1(2.4 \%)$ & $4(23.5 \%)$ & $5(8.5 \%)$ \\
\hline 1 a 4 anos & $6(14.3 \%)$ & $5(29.4 \%)$ & $11(18.6 \%)$ \\
\hline 5 a 10 anos & $23(54.8 \%)$ & $3(17.6 \%)$ & $26(44.1 \%)$ \\
\hline 11 a 15 anos & $6(14.3 \%)$ & $1(5.9 \%)$ & $7(11.9 \%)$ \\
\hline 16 a 20 anos & $4(9.5 \%)$ & $1(5.9 \%)$ & $5(8.5 \%)$ \\
\hline Mais de 20 anos & $2(4.8 \%)$ & $3(17.6 \%)$ & $5(8.5 \%)$ \\
\hline \multicolumn{4}{|l|}{ Nível máximo de titulação } \\
\hline Graduado & $1(2.4 \%)$ & $5(29.4 \%)$ & $6(10.2 \%)$ \\
\hline Especialista & $23(54.8 \%)$ & $8(47.1 \%)$ & $31(52.5 \%)$ \\
\hline Mestre & $14(33.3 \%)$ & $4(23.5 \%)$ & $18(30.5 \%)$ \\
\hline Doutor & $4(9.5 \%)$ & $0(0.0 \%)$ & $4(6.8 \%)$ \\
\hline \multicolumn{4}{|l|}{ Quantidade de UTI em que atua } \\
\hline 1 & $14(33.3 \%)$ & $7(41.2 \%)$ & $21(35.6 \%)$ \\
\hline 2 & $21(50.0 \%)$ & $5(29.4 \%)$ & $26(44.1 \%)$ \\
\hline 3 & $4(9.5 \%)$ & $5(29.4 \%)$ & $9(15.3 \%)$ \\
\hline 4 & $3(7.1 \%)$ & $0(0.0 \%)$ & $3(5.1 \%)$ \\
\hline
\end{tabular}

Legenda: Tempo de formação e atuação em UTI. Estatística descritiva; UTI - Unidade de Terapia Intensiva. Fonte: Os autores (2021).

Referente à infecção por COVID-19, 24 profissionais $(40,6 \%)$ relataram terem sido infectados pelo vírus SARSCoV-2 (dentre esses 17 da rede municipal e 7 da rede estadual). No que diz respeito aos fatores de risco para COVID-19, 15 (23.7\%) profissionais informaram apresentar algum fator, que se encontram na Figura 1. 
Figura 1. Fatores de risco relatados pelos profissionais participantes do estudo

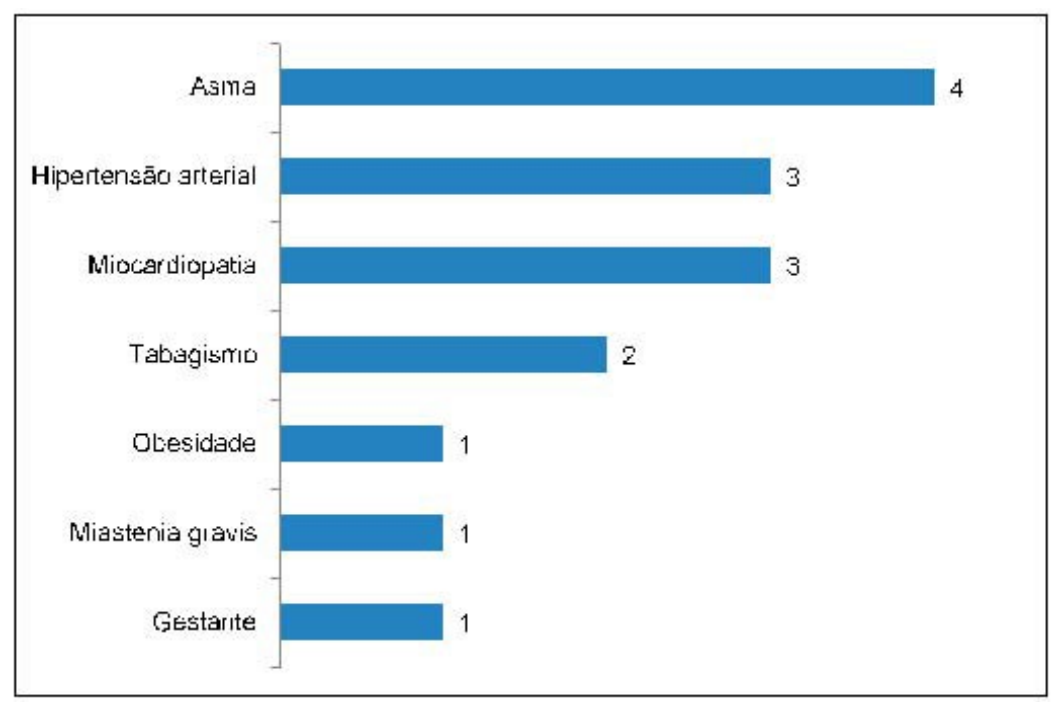

Legenda: Fatores de risco relatados. $\mathrm{N}=15$. Fonte: Os autores (2021).

Na Figura 2 encontram-se modificações na prática clínica de Fisioterapeutas Intensivistas durante a assistência ao paciente com COVID-19, sendo as mais relatadas pelos participantes do estudo a melhora no manejo da ventilação mecânica e a maior utilização de equipamentos de proteção individual.

Figura 2. Modificações na prática clínica

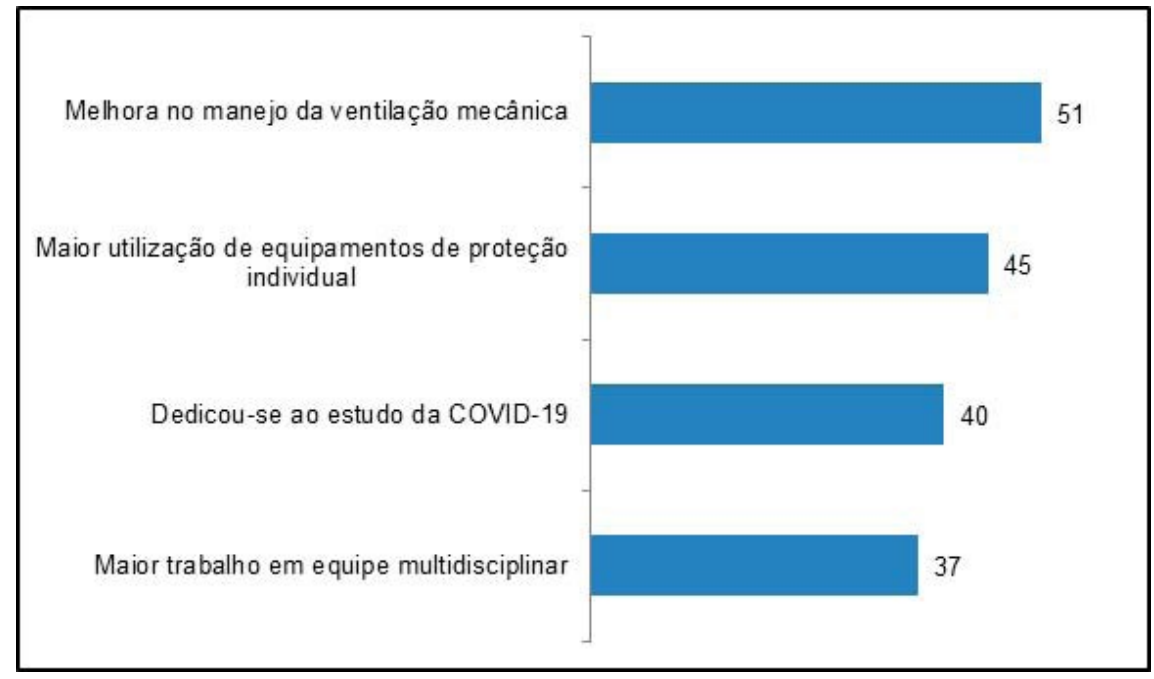

Legenda: Modificações na prática clínica durante assistência ao paciente com COVID-19. N=59.s. N=15. Fonte: Os autores (2021).

Encontram-se na Tabela 2, os principais desafios enfrentados pelos fisioterapeutas intensivistas durante assistência aos pacientes infectados com o novo coronavírus, sendo os mais citados: contexto completamente novo, pois o tratamento da doença ainda é pouco compreendido por 53 (89.8\%) profissionais, testemunhar experiências altamente dolorosas de pacientes por $45(76.3 \%)$ e carga de trabalho exaustiva para suprir a demanda populacional de atendimento de saúde relatada por 41 (69.5\%) profissionais. 


\begin{tabular}{|c|c|c|c|}
\hline & \multicolumn{2}{|c|}{ Hospital } & \multirow[b]{2}{*}{ Total } \\
\hline & Municipal & Estadual & \\
\hline Contexto completamente novo. & $39(92.9 \%)$ & $14(82.4 \%)$ & $53(89.8 \%)$ \\
\hline Carga de trabalho exaustiva. & $31(73.8 \%)$ & $10(58.8 \%)$ & $41(69.5 \%)$ \\
\hline Utilizou equipamentos geradores de aerossóis. & $11(26.2 \%)$ & $8(47.1 \%)$ & $19(32.2 \%)$ \\
\hline Testemunhou experiências dolorosas de pacientes. & $30(71.4 \%)$ & $15(88.2 \%)$ & $45(76.3 \%)$ \\
\hline Presenciou mortes em larga escala. & $27(64.3 \%)$ & $12(70.6 \%)$ & $39(66.1 \%)$ \\
\hline Necessitou realizar alocação de recursos. & $9(21.4 \%)$ & $1(5.9 \%)$ & $10(16.9 \%)$ \\
\hline
\end{tabular}

Legenda: Desafios enfrentados pelos Fisioterapeutas intensivistas na assistência a pacientes com o novo coronavírus. N=59. Fonte: Os autores (2021).

Estão descritos na Tabela 3 os resultados referentes a situações vivenciadas por Fisioterapeutas intensivistas durante a Pandemia Covid-19, sendo os mais relatados pelos profissionais municipais o afastamento dos familiares e a discriminação, e pelos fisioterapeutas estaduais o afastamento dos familiares e o desencadeamento/ intensificação de ansiedade.

Tabela 3. Situações vivenciadas por Fisioterapeutas Intensivistas

\begin{tabular}{lccc}
\hline & \multicolumn{2}{c}{ Hospital } & \multicolumn{1}{c}{ Total } \\
\cline { 2 - 3 } & Municipal & Estadual & $27(45.8 \%)$ \\
\hline Discriminação contra profissionais de saúde. & $20(47.6 \%)$ & $7(41.2 \%)$ & $45(76.3 \%)$ \\
Teve que se afastar dos familiares. & $\mathbf{3 3 ( 7 8 . 6 \% )}$ & $\mathbf{1 2 ( 7 0 . 6 \% )}$ & $3(5.1 \%)$ \\
Expulsão de transportes públicos. & $1(2.4 \%)$ & $2(11.8 \%)$ & $28(47.5 \%)$ \\
$\begin{array}{l}\text { Desencadeamento ou a intensificação de sintomas de } \\
\text { ansiedade. }\end{array}$ & $\mathbf{1 8 ( 4 2 . 9 \% )}$ & $\mathbf{1 0 ( 5 8 . 8 \% )}$ & $15(25.4 \%)$ \\
Perda dos colegas da equipe. & $\mathbf{6 ( 1 4 . 3 \% )}$ & $\mathbf{9 ( 5 2 . 9 \% )}$ & \\
\hline
\end{tabular}

Legenda: Situações vivenciadas por Fisioterapeutas intensivistas durante a Pandemia COVID-19. N=59. Estatística descritiva. Fonte: Os autores (2021).

Com relação a segurança para atender pacientes com Covid-19, a maioria 55 (93.2\%) dos profissionais relataram que, nas primeiras admissões de pacientes infectados por Covid-19 não se sentiam seguros durante os atendimentos e apenas 4 (6.8\%) participantes relataram se sentir seguros.

\section{Discussão}

O objetivo desse estudo foi verificar as modificações na prática clínica de Fisioterapeutas Intensivistas durante a Pandemia de COVID-19, bem como identificar os desafios enfrentados para assistência à população de pacientes críticos infectados pelo vírus SARS-CoV-2. No Boletim Epidemiológico do IntegraSUS $\frac{18}{2}$, atualizado no dia 12 de novembro de 2021, na cidade de Fortaleza foram confirmados 258.497 casos e 9.802 óbitos. No dia 8 de novembro, o Ministério da Saúde ${ }^{19}$ confirmou 152.147 casos de Covid-19 entre profissionais de saúde, estando os Fisioterapeutas em sétimo lugar com 6.001 casos. No presente estudo, 40,6\% dos Fisioterapeutas relataram terem sido infectados por esta doença. 
Estudos relatam que a principal via de transmissão de COVID-19 é por meio de aerossol, estando os profissionais de saúde, dentre eles o fisioterapeuta, em maior risco de contaminação devido à exposição ocupacional durante $o$ atendimento a pacientes suspeitos ou confirmados. ${ }^{20,21}$ Além disso, estão constantemente expostos aos procedimentos geradores de aerossol, como intubação endotraqueal, extubação, ventilação não invasiva, tratamentos com nebulizador e broncoscopia. 20,22 Estudo realizado por Liu et al. ${ }^{16}$, com 30 profissionais de saúde da China infectados por Covid-19, relatou que as principais razões para a infecção destes no ambiente hospitalar foram as medidas ineficazes de desinfecção e proteção, a necessidade de contato mais próximo com pacientes em estado crítico e a fadiga excessiva, desencadeando queda na imunidade.

Dentre as modificações na prática clínica, os participantes informaram haver maior trabalho em conjunto com a equipe multidisciplinar. Sobre esse achado, sabe-se que a relação multiprofissional e interorganizacional deve ser priorizada no ambiente hospitalar com o intuito de fornecer um atendimento mais eficiente e de maior qualidade para os pacientes, voltado para as necessidades de cada individuo. ${ }^{16,22}$ Palacios-Ceña et al. ${ }^{17}$ também observou uma maior colaboração entre as diversas especialidades em prol do paciente em seu estudo com fisioterapeutas atuantes na pandemia por COVID-19.

Em nossa análise, $86.4 \%$ dos participantes relataram uma melhora no manejo da ventilação mecânica em pacientes com COVID-19. Em situações de calamidade pública, como o surto de SARS-COV-2, ocorre um aumento significativo da necessidade de atuação de Fisioterapeutas nas UTI, com a principal finalidade de manter uma ventilação adequada nestes pacientes, que muitas vezes necessitam de ventilação mecânica invasiva. $\underline{24}$ Nesse sentido, documento publicado pela Associação Brasileira de Fisioterapia Respiratória (ASSOBRAFIR) apresenta ao Fisioterapeuta algumas estratégias ventilatórias importantes para o manejo da ventilação mecânica, dentre elas o volume corrente ajustado inicialmente em $6 \mathrm{ml} / \mathrm{Kg}$, a driving pressure menor que $15 \mathrm{cmH} 2 \mathrm{O}$ e pressão platô menor ou igual a $30 \mathrm{cmH} 20.2^{5}$

Quanto aos desafios enfrentados durante a assistência ao paciente com COVID-19, nossos resultados revelam que $89.8 \%$ dos participantes relataram o en- frentamento de um contexto completamente novo, e $67,8 \%$ informaram necessidade de dedicar um maior tempo para estudar sobre a patologia, tendo em vista se tratar de uma doença ainda pouco compreendida. Diante desse cenário, Carvalho e Kundsin ${ }^{26}$ enfatizam o treinamento dos profissionais para o enfrentamento da covid-19. Nesse contexto, a ASSOBRAFIR publicou vários documentos com recomendações assistenciais com o objetivo principal de capacitar os profissionais no manejo dessa assistência. ${ }^{24,25} \mathrm{Na}$ China, os profissionais entrevistados por Liu et al. $(2020)^{16}$ necessitaram recorrer a experiências clínicas anteriores e ao aprendizado por meio da exploração, levando em consideração que o tratamento da COVID-19 não é bem reconhecido.

Ademais, a carga de trabalho exaustiva para suprir a demanda de atendimentos foi observada em nossos resultados bem como a maior utilização de EPI"S. Oliveira et al. $\frac{13}{}$, pontuam que a sobrecarga de trabalho pode ser um fator importante para o desencadeamento de problemas como o estresse excessivo, ansiedade e insegurança. No estudo de Palacios-Ceña et al. ${ }^{17}$, a carga de trabalho extenuante juntamente com a necessidade de passar longos períodos com EPIs foi um grande desafio pessoal e profissional, fato também observado por Liu et al. $\frac{16}{6}$ em estudo com Médicos e Enfermeiros de Hospitais da província de Hubei e por Oliveira et al (2021) 27 com profissionais de saúde de um hospital da região metropolitana do Rio de Janeiro.

Um resultado marcante observado no presente estudo foi o relato dos profissionais em testemunhar experiências altamente dolorosas de pacientes. Em um estudo de abordagem qualitativa desenvolvido por Paula et al. .28 , com objetivo de compreender as reações e sentimentos dos profissionais da linha de frente do atendimento ao paciente com suspeita de COVID-19 internado em um Hospital Universitário, demonstrou que estes vivenciam um número significativo de sofrimento e morte, enfatizaram ainda que eles acabam vinculando a morte de paciente com um fracasso terapêutico e não como parte da vida, podendo desencadear sintomas de ansiedade e angústia. Outro achado importante foi a intensificação de sintomas de ansiedade, depressão e estresse, bem como a necessidade de afastamento de familiares.

Com relação à segurança para atender pacientes com COVID-19, a maioria (93.2\%) dos profissionais relatou 
insegurança nas primeiras admissões de pacientes infectados por COVID-19. Resultado semelhante foi observado por Ditwiler et al. ${ }^{29}$, mostrando que a incerteza foi um sentimento vivenciado pelos entrevistados da pesquisa, que relacionaram esta experiência com a falta de evidências sobre a doença e sua transmissão, baixa disponibilidade de EPIs adequados e a necessidade de utilizá-los por longo período. Nossos resultados corroboram com o que foi discutido por outros estudos, que relataram a falta de confiança e de segurança durante os primeiros atendimentos com pacientes infectados pelo vírus SARS-COV-2.30,31

A pandemia de COVID-19 limitou a realização das entrevistas aos profissionais fisioterapeutas, por conta do risco iminente de contaminação os Hospitais em questão não permitiam a entrada de pessoas que não fossem os profissionais da linha de frente, sendo necessária a realização da coleta dos dados ocorrer por meio do formulário eletrônico Google Forms, reduzindo assim o tamanho da amostra. Outros fatores limitantes foi a não investigação das razões que desencadearam a infecção dos participantes da pesquisa e a ausência de um questionário validado para esta investigação.

No contexto pandêmico os Fisioterapeutas Intensivistas enfrentaram novos desafios e uma maior complexidade do seu trabalho em cenário hospitalar, exercendo papel fundamental ao longo de toda a internação do paciente em UTI. Sugere-se dessa forma a realização de mais estudos relacionados a atuação do Fisioterapeuta durante a pandemia de COVID-19.

\section{Conclusão}

As modificações na prática clínica mais relatadas pelos Fisioterapeutas que participaram da pesquisa foram a melhoria no manejo da ventilação mecânica em pacientes com COVID-19 e o maior trabalho em conjunto com a equipe multidisciplinar.

Os principais desafios relatados durante assistência ao paciente infectado por SARS-COV-2 foram um contexto completamente novo, carga de trabalho exaustiva, intensificação dos sintomas de ansiedade, testemunhar experiências altamente dolorosas de pacientes e insegurança durante os primeiros atendimentos.

\section{Contribuições dos autores}

Viana CO e Viana MCC participaram da concepção, delineamento, busca e análise estatística dos dados da pesquisa, interpretação dos resultados, redação do artigo científico e encaminhamento do artigo científico. Pombo CMN participou na coleta e análise de dados. Morais MCS e Dantas MMP participaram da redação, concepção e do delineamento.

\section{Conflitos de interesses}

Nenhum conflito financeiro, legal ou político envolvendo terceiros (governo, empresas e fundações privadas, etc.) foi declarado para nenhum aspecto do trabalho submetido (incluindo, mas não se limitando a subvenções e financiamentos, participação em conselho consultivo, desenho de estudo, preparação de manuscrito, análise estatística, etc.).

\section{Referências}

1. Xiao H, Zhang Y, Kong D, Li S, Yang N. The effects of social support on sleep quality of medical staff treating patients with coronavirus disease 2019 (COVID-19) in January and February 2020 in China. Med Sci Monit. 2020;26:e923549. https://doi. org/10.12659/MSM.923549

2. Yifan T, Ying L, Chunhong G, Jing S, Rong W, Zhenyu L, et al. Symptom cluster of ICU nurses treating COVID-19 pneumonia patients in Wuhan, China. J Pain Symptom Manage. 2020;60(1):4851.https://doi.org/10.1016/j.jpainsymman.2020.03.039

3. Pegado R, Silva-Filho E, Lima IND, Gualdi L. Coronavirus disease 2019 (COVID-19) in Brasil: information to physical therapists. Rev Assoc Bras. 2020;66(4):498-501. https://doi.org/10.1590/1806-9282.66.4.498

4. Guan WJ, Ni ZY, Hu Y, Liang WH, Ou CQ, He JX, et al. Clinical characteristics of coronavirus disease 2019 in China. N Engl J Med. 2020;382(18);1708-20. https://doi.org/10.1056/NEJMoa2002032

5. Thomas P, Baldwin C, Bissett B, Boden I, Gosselink R, Granger $C$, et al. Physiotherapy management for COVID-19 in the acute hospital setting: clinical practice recommendations. J Physiother. 2020;66(2):73-82. https://doi.org/10.1016/j.jphys.2020.03.011

6. Chen N, Zhou M, Dong X, Qu J, Gong F, Han Y, et al. Epidemiological and clinical characteristics of 99 cases of 2019 novel coronavirus pneumonia in Wuhan, China: a descriptive study. Lancet. 2020;395(10223):507-13. https://doi.org/10.1016/ S0140-6736(20)30211-7 
7. Zhou F, Yu T, Du R, Fan G, Liu Y, Liu Z, et al. Clinical course and risk factors for mortality of adult inpatients with COVID-19 in Wuhan, China: a retrospective cohort study. Lancet. 2020;395(10229):1054-62. https://doi.org/10.1016/S0140$6736(20) 30566-3$

8. World Health Organization (Suíça). Coronavirus disease 2019 (COVID-19) Situation Report 46. Genebra: World Health Organization; 2020. Disponível em: https://apps.who.int/iris/ handle/10665/331443

9. Rangachari P, Woods JL. Preserving organizational resilience, patient safety, and staff retention during COVID-19 requires a holistic consideration of the psychological safety of healthcare workers. Int J Environ Res Public Health. 2020;17(12):4267. https:// doi.org/10.3390/ijerph17124267

10. Resolução $n^{\circ} 402$ de 03 de agosto de 2011 (Brasil). Dispõe sobre disciplina a Especialidade Profissional Fisioterapia em Terapia Intensiva e dá outras providências. [Internet]. Brasília: Conselho Federal de Fisioterapia e Terapia Ocupacional; 2011. Disponível em: https://www.coffito.gov.br/nsite/?p=3165

11. Santuzzi CH, Scardua MJ, Reetz JB, Firme KS, Lira NO, Gonçalves WLS. Aspectos éticos e humanizados da fisioterapia na UTI: uma revisão sistemática. Fisioter Mov. 2013;26(2):415-22. https://doi.org/10.1590/S0103-51502013000200019

12. Righetti RF, Onoue MA, Politi FVA, Teixeira DT, Souza PND, Kondo CS, et al. Physiotherapy care of patients with coronavirus disease 2019 (COVID-19)-a Brazilian experience. Clinics. 2020;75:e2017. https://doi.org/10.6061/clinics/2020/e2017

13. Oliveira KT, Junior JLG, Camandoni VO, Sousa JF, Canteras JS, Lima JL, et al. Principais medidas tomadas para a mudança dos processos assistenciais durante a pandemia por COVID-19. Enferm Foco. 2020;11(1)235-38. https://doi.org/10.21675/2357707X.2020.v11.n1.ESP.3764

14. Ornell F, Halpern SC, Kessler FHP, Narvaez JCM. O impacto da pandemia de COVID-19 na saúde mental dos profissionais de saúde. Cad Saúde Pública. 2020;36(4):e00063520. https://doi. org/10.1590/0102-311X00063520

15. Ministério da Saúde (Brasil). Resolução n 466, de 12 de dezembro de 2012 [Internet]. Brasília: Comissão Nacional de Ética em Pesquisa (CONEP); 2012. Disponível em: https://www.ip.usp. br/site/wp-content/uploads/2019/09/9_CNS_466_12.pdf

16. Liu Q, Luo D, Haase JE, Guo Q, Wang XQ, Liu S, et al. The experiences of health-care providers during the COVID-19 crisis in China: a qualitative study. Lancet Glob Health. 2020;8(6):e790-e798. https://doi.org/10.1016/S2214109X(20)30204-7
17. Palacios-Ceña D, Fernández-de-Las-Peñas C, Palacios-Ceña M, de-la-Llave-Rincón Al, Florencio LL. Working on the frontlines of the COVID-19 pandemic: A qualitative study of physical therapists' experience in Spain. Phys Ther. 2021;101(4):pzab025. https://doi. org/10.1093/ptj/pzab025

18. Prefeitura de Fortaleza (Brasil). Informe Semanal COVID-19 [Internet]. Fortaleza: Secretaria Municipal da Saúde; 2021. Available from: https://ms.dados.sms.fortaleza.ce.gov.br/ InformesemanalCOVID19SE452021.pdf

19. Ministério da Saúde (Brasil). Doença pelo Novo Coronavírus: COVID-19 [Internet]. Brasília: Secretaria de Vigilância em Saúde; 2021. Available from: https://www.gov.br/saude/pt-br/media/ pdf/2021/outubro/18/boletim_epidemiologico_covid_84-final.pdf

20. Ng K, Poon BH, Puar THK, Quah JLS, Loh WJ, Wong YJ, et al. COVID-19 and the risk to health care workers: a case report. Ann Intern Med. 2020;172(11):766-67. https://doi.org/10.7326/L20$\underline{0175}$

21. Ran L, Chen X, Wang Y, Wu W, Zhang L, Tan X. Risk factors of healthcare workers with corona virus disease 2019: a retrospective cohort study in a designated hospital of Wuhan in China. Clin. 2020;71(16):2218-21. https://doi.org/10.1093/cid/ ciaa287

22. Heinzerling A, Stuckey MJ, Scheuer T, Xu K, Perkins KM, Resseger $\mathrm{H}$, et al. Transmission of COVID-19 to health care personnel during exposures to a hospitalized patient: Solano County, California, February 2020. MMWR Weekly Report. 2020;69(15):472-76. https://doi.org/10.15585/mmwr.mm6915e5

23. Silva ANM, Santos AP. Atuação do fisioterapeuta residente em infectologia no contexto da covid-19: possibilidades e desafios [Internet]. Cadernos ESP. 2020;14(1):152-55. https://cadernos.esp. ce.gov.br/index.php/cadernos/article/view/380

24. Martinez BP, Andrade FMD. Estratégias de mobilização e exercícios terapêuticos precoces para pacientes em ventilação mecânica por insuficiência respiratória aguda secundária a covid-19. ASSOBRAFIR Ciênc. 2020;11(S1):121-31. http://dx.doi. org/10.47066/2177-9333.AC20.covid19.012

25. Musumeci MM, Martinez BP, Nogueira IC, Alcanfor T. Recursos fisioterapêuticos utilizados em unidades de terapia intensiva para avaliação e tratamento das disfunções respiratórias de pacientes com COVID-19. ASSOBRAFIR Ciênc. 2020;11(S1):73-86. http:// dx.doi.org/10.47066/2177-9333.AC20.covid19.007

26. Carvalho ES, Kundsin A. Atuação do fisioterapeuta mediante a pandemia da COVID-19 em um hospital de referência no interior da Amazônia Legal. Rev. Eletrônica Acervo Saúde. 2021;13(2):e6435. https://doi.org/10.25248/reas.e6435.2021 
27. Oliveira ACS, Silva GF, França LCM, Vargas GSA, Firmino G. Percepção dos profissionais de saúde na pandemia por COVID-19: desafios e estratégias para prática profissional. Res Soc Dev. 2021;10(10):e350101018724. https://doi.org/10.33448/rsdv10i10.18724

28. Paula ACR, Carletto AGD, Lopes D, Ferreira JC, Tonini NS, Trecossi SPC. Reações e sentimentos dos profissionais de saúde no cuidado de pacientes hospitalizados com suspeita COVID-19. Rev Gaúcha Enferm. 2021;42(esp):e20200160. https://doi. org/10.1590/1983-1447.2021.20200160

29. Ditwiler RE, Swisher LL, Hardwick DD. Professional and ethical issues in United States acute care physical therapists treating patients with COVID-19: stress, walls, and uncertainty. Phys Ther. 2021;101(8):pzab122.https://doi.org/10.1093/ptj/pzab122
30. Hu D, Kong Y, Li W, Han Q, Zhang X, Zhu LX. Frontline nurses' burnout, anxiety, depression, and fear statuses and their associated factors during the COVID-19 outbreak in Wuhan, China: A large-scale cross-sectional study. EClinicalMedicine 2020;24:100424. https://doi.org/10.1016/j.eclinm.2020.100424

31. Zhang C, Yang L, Liu S, Ma S, Wang Y, Cai Z, et al. Survey of insomnia and related social psychological factors among medical staff involved in the 2019 novel Coronavirus disease outbreak. Front Psychiatry. 2020;11:306. https://doi.org/10.3389/ fpsyt.2020.00306 